\title{
ANEMIA Y ESTADO NUTRICIONAL EN LACTANTES DE DOS A CINCO MESES ATENDIDOS EN ESTABLECIMIENTOS DEL MINISTERIO DE SALUD DEL PERÚ, 2012
}

\author{
Guillermo Gómez-Guizado1,a, Oscar Munares-García ${ }^{1,2, b}$
}

\begin{abstract}
RESUMEN
Objetivos. Determinar las características de los niveles de anemia en lactantes de dos a cinco meses de edad atendidos en los establecimientos del Ministerio de Salud del Perú durante el año 2012. Materiales y métodos. Estudio transversal utilizando la base de datos del Sistema de Información del Estado Nutricional del Centro Nacional de Alimentación y Nutrición del Instituto Nacional de Salud del Perú del año 2012. Se analizaron los registros de 7513 lactantes de dos a cinco meses de edad atendidos en puestos, centros y hospitales del Ministerio de Salud de las 25 regiones del Perú. Se utilizaron estadísticas descriptivas y de regresión logística. Resultados. El 10,2\% (IC 95\%: 9,5-10,9) de los lactantes presentaron anemia, con diferencias en las frecuencias según los meses de edad $(p<0,001)$, altitud a nivel del mar $(p<0,001)$, regiones $(p<0,001)$ y tipo de establecimiento $(p<0,001)$. En el análisis multivariable se encontraron como factores asociados con tener anemia la edad de 4 meses (OR 1,2; IC 95\%: 1,0-1,5) y 5 meses (OR 1,4; IC 95\%: 1,2-1,6), presentar obesidad (OR 0,7; IC 95\%: 0,5-1,0) y desnutrición crónica (OR 1,3; IC 95\%: 1,0-1,6). Conclusiones. Se evidencio la presencia de anemia y desnutrición crónica antes de los 6 meses de edad y que la probabilidad de tener anemia se incrementa conforme aumenta la edad y está asociada a algunos componentes del estado nutricional.
\end{abstract}

Palabras clave: Anemia; Estado nutricional; Lactante; Desnutrición (fuente: DECS/BIREME).

\section{ANEMIA AND NUTRITIONAL STATUS OF INFANTS FROM TWO TO FIVE MONTHS OF AGE TREATED IN FACILITIES OF THE MINISTRY OF HEALTH OF PERU, 2012}

\begin{abstract}
Objectives. Determine the characteristics of the levels of anemia in infants from two to five months of age treated in facilities of the Ministry of Health of Peru during 2012. Materials and methods. This cross-sectional study uses the nutritional status information system of the National Center for Food and Nutrition, National Institute of Health of Peru in 2012. Records of 7,513 infants from two to five months of age treated in small outpatient clinics, centers and hospitals of the Ministry of Health of Peru's 25 regions were analyzed. Descriptive statistics and logistic regression techniques were used for data analysis. Results. $10.2 \%$ (95\% CI 9.5-10.9) of the infants had anemia, with frequency differences by month of age $(p<0.001)$, altitude, $(p<0.001)$ regions $(p<0.001)$ and type of facility $(p<0.001)$. In the multivariate analysis, factors associated with anemia were: age of 4 months (OR 1.2; 95\% $\mathrm{Cl} 1.0-1.5)$, age of 5 months (OR 1.4; 95\% $\mathrm{Cl} 1.2-$ 1.6), obesity (OR $0.7 ; 95 \% \mathrm{Cl} 0.5-1.0$ ) and chronic malnutrition (OR 1.3; 95\% Cl 1.0-1.6). Conclusions. The presence of anemia and chronic malnutrition before 6 months of age was evidenced, as well as that the probability of anemia increases with age and is associated with some components of nutritional status.
\end{abstract}

Key words: Anemia; Nutritional status; Infant; Malnutrition (source: MeSH/NLM).

\footnotetext{
Centro Nacional de Alimentación y Nutrición, Instituto Nacional de Salud. Lima, Perú.

Facultad de Medicina, Universidad Nacional Mayor de San Marcos. Lima, Perú.

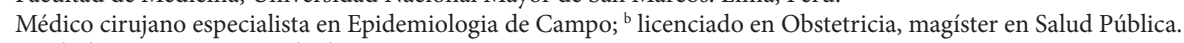

Recibido: 14-10-13 Aprobado: 28-05-14
}

Citar como: Gómez-Guizado G, Munares-García O. Anemia y estado nutricional en lactantes de dos a cinco meses atendidos en establecimientos del Ministerio de Salud del Perú, 2012. Rev Peru Med Exp Salud Publica. 2014;31(3):487-93. 


\section{INTRODUCCIÓN}

Según información de la Organización Mundial de la Salud (OMS) (1), el $90 \%$ de la anemia en el mundo se debe a la deficiencia de hierro (DH). En América del Sur y Centroamérica, la anemia afecta aproximadamente a la mitad de las gestantes y niños ${ }^{(2)}$. La DH constituye un importante problema de salud pública, y que tiene a los lactantes como la población de mayor riesgo, por el acelerado crecimiento en esta etapa y la dieta insuficiente y de baja biodisponibilidad de hierro ${ }^{(3)}$, por lo que, en niños menores de 3 años, más del $95 \%$ de la $\mathrm{DH}$ es de índole dietética ${ }^{(4,5)}$.

Un estudio realizado en Argentina ${ }^{(6)}$ evidenció altas prevalencias de anemia ya desde los 6 meses, aun en niños de término con peso adecuado al nacer, lo cual sugiere que la anemia podría ser favorecida por factores durante el embarazo, el periodo perinatal, o en ambos. En el Perú, el $63,7 \%$ de lactantes a esta edad son anémicos, de los cuales prácticamente la mitad son anemias leves y la otra mitad anemias moderadas ${ }^{(7)}$, resultando obvio que estas últimas han iniciado la anemia antes de los 6 meses, y mucho antes la DH. Por otro lado, la desnutrición crónica en menores de 6 meses, según los estándares de la OMS, fue de $13,3 \%$ en el $2012{ }^{(7)}$, pero en años previos (2004-2008) la proporción fue mayor e incluso solo superada por Guatemala y Haití en este grupo etario ${ }^{(8)}$.

El límite normal para la hemoglobina en niños de seis meses a seis años es de $11,0 \mathrm{~g} / \mathrm{dL}^{(9,10)}$ y de $9,5 \mathrm{~g} / \mathrm{dL}$ entre los 2 a 5 meses ${ }^{(11)}$, cifras menores son consideradas como anemia. Un estudio realizado en Argentina determinó una prevalencia de anemia $(\mathrm{Hb}<10,3 \mathrm{~g} / \mathrm{dL})$ a los 4 meses de $20,6 \%$ y a los 5 meses de $30 \%$ (2). En Brasil, se encontró una frecuencia de anemia $(\mathrm{Hb}<10,3 \mathrm{~g} / \mathrm{dL})$ de $20 \%$ para los niños menores de 6 meses ${ }^{(12)}$. Actualmente, no se tiene una precisión de la prevalencia de anemia en menores de seis meses en el Perú, por lo que el objetivo de la presente investigación fue determinar las características de la anemia y el estado nutricional en lactantes de dos a cinco meses atendidos en los establecimientos del Ministerio de Salud en el Perú.

\section{MATERIALES Y MÉTODOS}

\section{DISEÑO Y POBLACIÓN DE ESTUDIO}

Estudio de tipo trasversal, se realizó un análisis de la base de datos del Sistema de Información del Estado Nutricional (SIEN), del Centro Nacional de Alimentación y Nutrición (CENAN) del Instituto Nacional de Salud del Perú para el periodo de enero a diciembre del año 2012.
EI SIEN registra solo datos para evaluación nutricional y hemoglobina de niños menores de cinco años, dentro de su control, atendidos en 1852 establecimientos del Ministerio de Salud del Perú. Se realizó un censo de todos los registros del sistema SIEN, y de ellos se incluyeron registros de niños entre los dos y cinco meses, se excluyeron registros vacíos o con inconsistencias en cualquiera de las variables estudiadas, de lo cual resultaron 7513 registros.

\section{PROCESAMIENTO DE DATOS}

De acuerdo con la norma de suplementación preventiva de hierro en niños menores de tres años ${ }^{(13)}$, el profesional que atiende solicita el dosaje de hemoglobina, una vez obtenido, se informa el resultado en la historia clínica y en el formato de registro SIEN por el personal que realiza la consulta ${ }^{(10)}$. Luego, los formatos pasan al punto de digitación, pudiendo ser el mismo establecimiento u otro, este incorpora al sistema SIEN la información que después es consolidada mensualmente. Cada punto de digitación realiza el control de calidad sobre registros incompletos $y$ datos inconsistentes. Los informes de hemoglobina ajustada a la altura son realizados automáticamente por el sistema SIEN ${ }^{(14)}$. La medición de hemoglobina en los establecimientos de salud fue mediante el hemoglobinómetro portátil o por la centrífuga para hematocrito. Es preciso mencionar que el criterio para realizar la medición de la hemoglobina antes de los 6 meses de edad se desconoce, asimismo, si la decisión de la prueba fue solicitada por los padres o el personal de salud.

\section{VARIABLES}

Los niveles de hemoglobina se determinaron en $\mathrm{g} / \mathrm{dL}$, y correspondió al último dato registrado. Para la determinación de la anemia se tomó como punto de corte la $\mathrm{Hb}<9,5 \mathrm{~g} / \mathrm{dL}$ según los criterios de la OMS ${ }^{(11)}$. Se determinó los meses de edad de los lactantes, sexo, altitud sobre el nivel del mar, región de residencia, tipo de establecimiento de salud y estado nutricional, determinado por el z score, según los estándares de la OMS del 2006 para peso/talla (desnutrición aguda, sobrepeso u obesidad), talla/edad (desnutrición crónica) y peso/edad (desnutrición global), el cual se aplicó según los criterios de periodicidad establecidos en la norma técnica para el control del crecimiento y desarrollo de la niña y el niño menor de cinco años, de uso obligatorio en los establecimientos del Ministerio de Salud (15).

\section{ANÁLISIS DE DATOS}

Se determinó la normalidad de los datos con la prueba de Kolmorogov-Smirnov y en cifras menores a 30 
registros se analizó con la prueba Shapiro-Wilk. Se aplicó distribución de frecuencias, media, desviación estándar y percentiles 25,50 (mediana) y 75 ; así mismo, para la determinación de las diferencias en las medias de las cifras de hemoglobina según variables seleccionadas, se aplicó la prueba $U$ de Mann Whitney y Kruscal-Wallis. Para la determinación de las diferencias en las variables cualitativas se aplicó chi cuadrado y la prueba exacta de Fisher cuando las celdas fueron menores a cinco unidades. En el análisis bivariado se aplicó la razón de productos cruzados (ORc) con sus intervalos de confianza al 95\% (IC 95\%) y regresión logística multivariable, bajo el método introducir (ORa). Se empleó el programa estadístico SPSS 20.

\section{CONSIDERACIONES ÉTICAS}

La información fue manejada de forma confidencial, la base de datos solo incluyo como dato de identificación el número de historia clínica; no fueron registrados otros datos como el nombre, la dirección, u otros de identificación personal.

\section{RESULTADOS}

En todos los casos las cifras no presentaron una distribución normal, para la prueba de Kolmorogov-Smirnov y Shapiro Wilk. El $51,1 \%$ de la población (3842) fue de sexo masculino. El $25 \%$ presentó valores de $\mathrm{Hb}$ de $10,2 \mathrm{~g} / \mathrm{dL}$ o menos y la mitad de la población $11,2 \mathrm{~g} / \mathrm{dL}$, con respecto a la altitud, la mediana de hemoglobina $\left(P_{50}\right)$ se incrementa a mayor altitud, siendo de $11,0 \mathrm{~g} / \mathrm{dL}$ para los que residen entre los 2 a 1000 metros de altitud y de $13,0 \mathrm{~g} / \mathrm{dL}$ para los que residen entre 4001 a 4801 metros. Las regiones con lactantes con una mediana de hemoglobina mayor fueron: Callao, Cusco, Huancavelica, La Libertad y Tacna $(12,0 \mathrm{~g} / \mathrm{dL})$; la región de Apurímac presentó la mediana más baja con 10,4 g/dL (Tabla 1).

La proporción global de anemia fue de 10,2\% (IC 95\%: $9,5-10,9)$, con diferencias en las frecuencias según los meses de edad $(p<0,001)$, altitud a nivel del mar $(p<0,001)$, regiones $(p<0,001)$ y tipo de establecimiento $(p<0,001)$. No se observaron diferencias en los porcentajes de anemia por sexo. Los lactantes que residen entre los 3001 a 4000 metros de altitud tienen mayor porcentaje de anemia (13,7\%; IC 95\%: 12,3-15,1). Loreto presentó mayor porcentaje de lactantes con anemia $(27,0 \%)$. Los casos de anemia se presentaron con más frecuencia en los puestos de salud (Tabla 2).

El promedio de lactantes con desnutrición aguda fue del 0,7\% (IC 95\%: 0,5-0,8). Las cifras más altas fueron encontradas en las regiones de Tacna;
Tabla 1. Niveles de hemoglobina $(\mathrm{g} / \mathrm{dL})$ en lactantes de dos a cinco meses atendidos en establecimientos del Ministerio de Salud del Perú, 2012

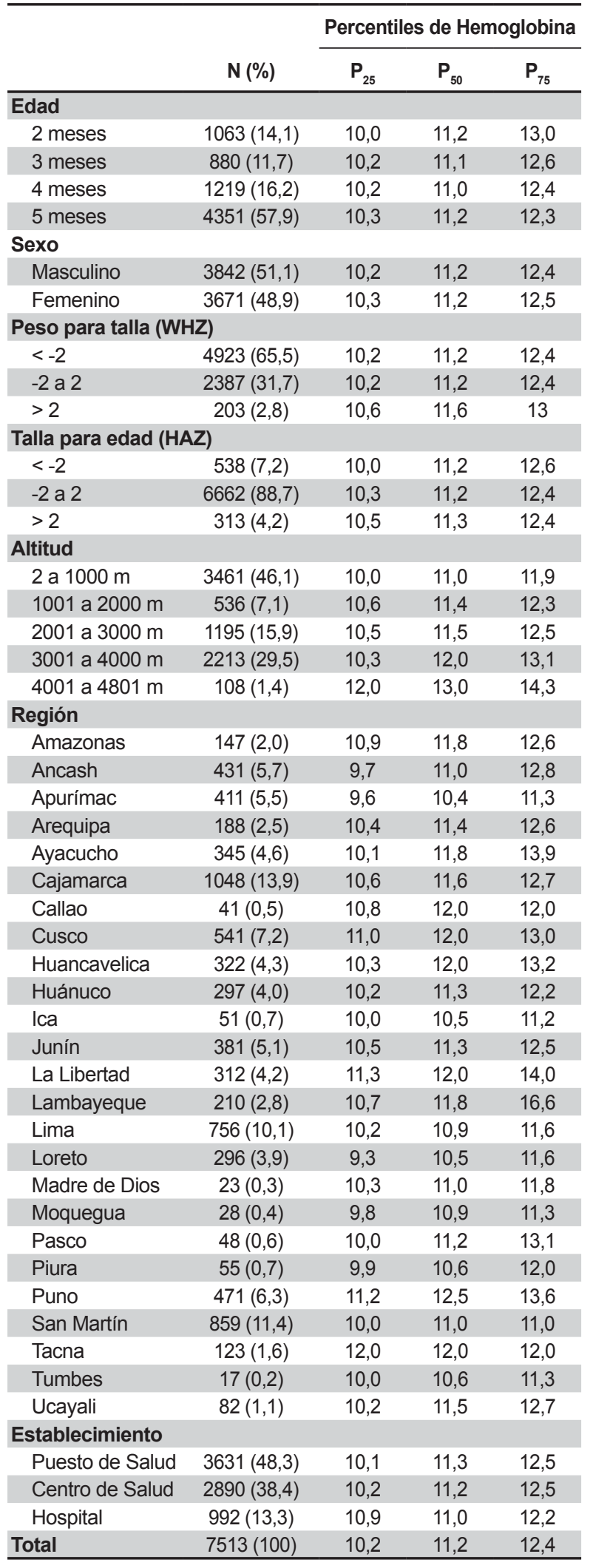


Huánuco; Huancavelica, Ica y Arequipa. Con respecto al sobrepeso, se encontró una cifra total de 10,1\% (IC 95\%: 8,0-12,2); siendo el mayor porcentaje a los 4 meses. Callao e Ica presentaron las frecuencias más altas de sobrepeso. Con respecto a la obesidad se encontró una proporción de 2,6\% (IC 95\%: 0,4-4,8), la frecuencia de obesidad es menor conforme aumenta los meses de edad, siendo de $3,7 \%$ para los dos meses y de 2,1\% para los cinco meses. La región de Callao presentó la mayor frecuencia de obesidad $(14,6 \%)$. Los centros de salud, fueron los lugares donde se presentó mayor frecuencia de casos. Las variables de sexo y altitud presentan diferencias significativas $(p<0,05)$, como también edad, regiones de residencia y tipo de establecimiento de salud $(p<0,001)$ (Tabla 2).

Tabla 2. Distribución de anemia y estado nutricional en lactantes de dos a cinco meses atendidos en establecimientos del Ministerio de Salud del Perú, 2012

\begin{tabular}{|c|c|c|c|c|c|c|c|c|c|c|c|c|c|c|c|c|}
\hline & \multicolumn{2}{|c|}{ Anemia } & \multirow[t]{2}{*}{$p$} & \multicolumn{2}{|c|}{$\begin{array}{c}\text { Desnutrición } \\
\text { aguda }\end{array}$} & \multicolumn{2}{|c|}{ Sobrepeso } & \multicolumn{2}{|c|}{ Obesidad } & \multirow[t]{2}{*}{$p$} & \multicolumn{2}{|c|}{$\begin{array}{c}\text { Desnutrición } \\
\text { crónica }\end{array}$} & \multicolumn{3}{|c|}{$\begin{array}{c}\text { Desnutrición } \\
\text { global }\end{array}$} & \multirow[t]{2}{*}{ p } \\
\hline & $\%$ & (IC 95\%) & & $\%$ & (IC 95\%) & $\%$ & (IC 95\%) & $\%$ & (IC 95\%) & & $\%$ & (IC 95\%) & & $\%$ & (IC 95\%) & \\
\hline \multicolumn{17}{|l|}{ Edad } \\
\hline 2 meses & 11,9 & $(6,2-17,5)$ & $* *$ & 0,7 & $(0,0-6,7)$ & 9,8 & $(4,1-15,5)$ & 3,7 & $(0,0-9,6)$ & $* *$ & 7,4 & $(1,6-13,2)$ & $* *$ & 1,5 & $(0,0-7,5)$ & \\
\hline 3 meses & 10,1 & $(3,8-16,4)$ & & 0,8 & $(0,0-7,4)$ & 10,8 & $(4,6-17,0)$ & 3,4 & $(0,0-9,9)$ & & 6,7 & $(0,3-13,1)$ & & 1,6 & $(0,0-8,1)$ & \\
\hline 4 meses & 9,6 & $(4,3-14,9)$ & & 0,8 & $(0,0-6,4)$ & 11,2 & $(5,9-16,5)$ & 2,9 & $(0,0-8,4)$ & & 5,7 & $(0,2-11,1)$ & & 1,4 & $(0,0-7,0)$ & \\
\hline 5 meses & 10,0 & $(7,1-12,8)$ & & 0,6 & $(0,0-3,6)$ & 9,7 & $(6,9-12,5)$ & 2,1 & $(0,0-5,1)$ & & 7,2 & $(4,4-10,1)$ & & 1,3 & $(0,0-4,3)$ & \\
\hline \multicolumn{17}{|l|}{ Sexo } \\
\hline Masculino & 10,9 & $(9,9-11,9)$ & & 0,8 & $(0,5-1,1)$ & 9,4 & $(6,4-12,4)$ & 2,2 & $(0,0-5,3)$ & * & 8,2 & $(7,3-9,1)$ & ** & 1,6 & $(1,2-2,0)$ & \\
\hline Femenino & 9,4 & $(8,5-10,3)$ & & 0,5 & $(0,3-0,8)$ & 10,8 & $(7,8-13,9)$ & 3,0 & $(0,0-6,2)$ & & 5,6 & $(4,9-6,4)$ & & 1,2 & $(0,8-1,5)$ & \\
\hline \multicolumn{17}{|l|}{ Altitud } \\
\hline 2 a $1000 \mathrm{~m}$ & 9,0 & $(8,0-9,9)$ & $* *$ & 0,8 & $(0,5-1,1)$ & 10,5 & $(7,4-13,7)$ & 2,9 & $(0,0-6,2)$ & * & 4,7 & $(4,0-5,4)$ & ** & 1,2 & $(0,8-1,5)$ & $* *$ \\
\hline 1001 a 2000 m & 5,0 & $(3,2-6,9)$ & & 0,4 & $(0,0-0,9)$ & 11,6 & $(3,6-19,5)$ & 2,6 & $(0,0-11,0)$ & & 5,0 & $(3,2-6,9)$ & & 0,6 & $(0,0-1,2)$ & \\
\hline 2001 a 3000 m & 10,0 & $(8,3-11,7)$ & & 0,1 & $(0,0-0,2)$ & 10,4 & $(5,0-15,7)$ & 2,9 & $(0,0-8,5)$ & & 8,7 & $(7,1-10,3)$ & & 1,3 & $(0,0,7-2)$ & \\
\hline 3001 a 4000 m & 13,7 & $(12,3-15,1)$ & & 0,8 & $(0,4-1,2)$ & 9,1 & $(5,1-13,1)$ & 2 & $(0,0-6,2)$ & & 9,6 & $(8,4-10,9)$ & & 1,8 & $(1,2-2,3)$ & \\
\hline 4001 a 4801 m & 4,6 & $(0,7-8,6)$ & & 0,9 & $(0,0-2,7)$ & 7,4 & $(0,0-25,6)$ & 0,9 & $(0,0-19,7)$ & & 15,7 & $(8,9-22,6)$ & & 4,6 & $(0,7-8,6)$ & \\
\hline \multicolumn{17}{|l|}{ Región } \\
\hline Amazonas & 9,5 & $(4,8-14,3)$ & ** & 0,7 & $(0,0,6-2)$ & 7,5 & $(0,0-23,0)$ & 3,4 & $(0,0-19,3)$ & $* *$ & 4,8 & $(1,3-8,2)$ & $* *$ & 0,7 & $(0,0-2,0)$ & * \\
\hline Ancash & 21,1 & $(17,3-25,0)$ & & 0,7 & $(0,0-1,5)$ & 7,9 & $(0,0-16,9)$ & 1,6 & $(0,0-11,0)$ & & 10,7 & $(7,8-13,6)$ & & 2,8 & $(1,2-4,3)$ & \\
\hline Apurímac & 20,4 & $(16,5-24,3)$ & & 0,2 & $(0,0-0,7)$ & 9,0 & $(0,0-18,2)$ & 0,7 & $(0,0-10,4)$ & & 9,7 & $(6,9-12,6)$ & & 1,2 & $(0,2-2,3)$ & \\
\hline Arequipa & 6,9 & $(3,3-10,5)$ & & 1,1 & $(0,0-2,5)$ & 6,4 & $(0,0-20,2)$ & 3,2 & $(0,0-17,3)$ & & 5,3 & $(2,1-8,5)$ & & 2,1 & $(0,1-4,2)$ & \\
\hline Ayacucho & 18,0 & $(13,9-22,0)$ & & 0,9 & $(0,0-1,8)$ & 7,8 & $(0,0-18,0)$ & 2,0 & $(0,0-12,5)$ & & 9,9 & $(6,7-13,0)$ & & 3,2 & $(1,3-5,0)$ & \\
\hline Cajamarca & 7,4 & $(5,9-9,0)$ & & 0,4 & $(0,0-0,8)$ & 12,4 & $(6,7-18,1)$ & 2,9 & $(0,0-8,8)$ & & 9,0 & $(7,2-10,7)$ & & 1,4 & $(0,7-2,2)$ & \\
\hline Callao & 7,3 & $(0,0-15,3)$ & & 0 & $\ldots$ & 19,5 & $(0,0-47,0)$ & 14,6 & $(0,0-42,9)$ & & 17,1 & $(5,6-28,6)$ & & 2,4 & $(0,0-7,2)$ & \\
\hline Cusco & 5,2 & $(3,3-7,0)$ & & 0,7 & $(0,0-1,5)$ & 7,0 & $(0,0-15,1)$ & 1,1 & $(0,0-9,5)$ & & 6,8 & $(4,7-9,0)$ & & 2,4 & $(1,1-3,7)$ & \\
\hline Huancavelica & 14,3 & $(10,5-18,1)$ & & 1,2 & $(0,0-2,5)$ & 9,0 & $(0,0-19,4)$ & 3,1 & $(0,0-13,9)$ & & 9,3 & $(6,1-12,5)$ & & 0,9 & $(0,0-2,0)$ & \\
\hline Huánuco & 10,1 & $(6,7-13,5)$ & & 1,3 & $(0,0-2,7)$ & 10,4 & $(0,0-21,2)$ & 1,7 & $(0,0-13,0)$ & & 6,4 & $(3,6-9,2)$ & & 0,7 & $(0,0-1,6)$ & \\
\hline Ica & 14,8 & $(7,1-22,6)$ & & 1,2 & $(0,0-3,6)$ & 14,8 & $(0,0-34,9)$ & 1,2 & $(0,0-22,9)$ & & 8,6 & $(2,5-14,8)$ & & 1,2 & $(0,0-3,6)$ & \\
\hline Junín & 8,1 & $(5,4-10,9)$ & & 0,8 & $(0,0-1,7)$ & 8,4 & $(0,0-18,0)$ & 2,6 & $(0,0-12,5)$ & & 10 & $(7,0-13,0)$ & & 1,3 & $(0,2-2,5)$ & \\
\hline La Libertad & 1,0 & $(0,0-2,0)$ & & 0,6 & $(0,0-1,5)$ & 14,7 & $(4,5-25,0)$ & 4,2 & $(0,0-15,0)$ & & 7,1 & $(4,2-9,9)$ & & 1,0 & $(0,0-2,0)$ & \\
\hline Lambayeque & 4,8 & $(1,9-7,6)$ & & 0,5 & $(0,0-1,4)$ & 11 & $(0,0-23,7)$ & 7,6 & $(0,0-20,6)$ & & 5,7 & $(2,6-8,9)$ & & 0,5 & $(0,0-1,4)$ & \\
\hline Lima & 7,7 & $(5,8-9,6)$ & & 0,8 & $(0,2-1,4)$ & 10,7 & $(4,0-17,5)$ & 4,0 & $(0,0-11,0)$ & & 2,2 & $(1,2-3,3)$ & & 0,3 & $(0,0-0,6)$ & \\
\hline Loreto & 27,0 & $(22,0-32,1)$ & & 0,3 & $(0,0-1,0)$ & 8,1 & $(0,0-19,0)$ & 2,4 & $(0,0-13,6)$ & & 9,5 & $(6,1-12,8)$ & & 2,4 & $(0,6-4,1)$ & \\
\hline Madre de Dios & 13,0 & $(0,0-26,8)$ & & 0 & $\ldots$ & 13 & $(0,0-51,2)$ & 4,3 & $(0,0-44,3)$ & & 0 & $\cdots$ & & 0 & $\ldots$ & \\
\hline Moquegua & 14,3 & $(1,3-27,2)$ & & 0 & $\ldots$ & 14,3 & $(0,0-48,6)$ & 0 & & & 7,1 & $(0,0-16,7)$ & & 0 & $\ldots$ & \\
\hline Pasco & 14,6 & $(4,6-24,6)$ & & 0 & $\ldots$ & 8,3 & $(0,0-35,4)$ & 0 & ... & & 4,2 & $(0,0-9,8)$ & & 0 & ... & \\
\hline Piura & 12,7 & $(3,9-21,5)$ & & 0 & $\ldots$ & 7,3 & $(0,0-32,7)$ & 7,3 & $(0,0-32,7)$ & & 7,3 & $(0,4-14,1)$ & & 1,8 & $(0,0-5,3)$ & \\
\hline Puno & 7,4 & $(5,1-9,8)$ & & 0,8 & $(0,0-1,7)$ & 8,9 & $(0,3-17,5)$ & 1,1 & $(0,0-10,0)$ & & 5,9 & $(3,8-8,1)$ & & 1,9 & $(0,7-3,1)$ & \\
\hline San Martín & 6,3 & $(4,7-7,9)$ & & 0,5 & $(0,0-0,9)$ & 12,1 & $(5,8-18,4)$ & 2,7 & $(0,0-9,3)$ & & 3,5 & $(2,3-4,7)$ & & 0,6 & $(0,1-1,1)$ & \\
\hline Tacna & 1,6 & $(0,0-3,9)$ & & 1,6 & $(0,0-3,9)$ & 10,6 & $(0,0-27,3)$ & 0,8 & $(0,0-18,4)$ & & 0,8 & $(0,0-2,4)$ & & 1,6 & $(0,0-3,9)$ & \\
\hline Tumbes & 17,6 & $(0,0-35,8)$ & & 0 & $\ldots$ & 5,9 & $(0,0-52,0)$ & 0 & $\ldots$ & & 0 & & & 0 & 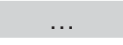 & \\
\hline Ucayali & 8,5 & $(2,5-14,6)$ & & 0 & $\ldots$ & 11 & $(0,0-31,4)$ & 0 & $\ldots$ & & 8,5 & $(2,5-14,6)$ & & 1,2 & $(0,0-3,6)$ & \\
\hline \multicolumn{17}{|l|}{ Establecimiento } \\
\hline Puesto de Salud & 11,5 & $(8,4-14,5)$ & ** & 0,8 & $(0,0-4,0)$ & 10,7 & $(7,7-13,8)$ & 2,5 & $(0,0-5.7)$ & $* \star$ & 5,9 & $(2,3-9,4)$ & ** & 1,6 & $(0,0-4,8)$ & \\
\hline Centro de Salud & 9,0 & $(5,6-12,5)$ & & 0,6 & $(0,0-4,2)$ & 9,8 & $(6,3-13,3)$ & 3,1 & $(0,0-6.7)$ & & 4,4 & $(0,0-10,5)$ & & 1,1 & $(0,0-4,7)$ & \\
\hline Hospital & 8,8 & $(2,8-14,7)$ & & 0,5 & $(0,0-6,7)$ & 8,7 & $(2,7-14,6)$ & 1,5 & $(0,0-7.7)$ & & 6,9 & $(4,8-9,1)$ & & 1,5 & $(0,0-7,7)$ & \\
\hline Total & 10,2 & $(9,5-10,9)$ & & 0,7 & $(0,5-0,8)$ & 10,1 & $(8,0-12,2)$ & 2,6 & $(0,4-4,8)$ & & 6,9 & $(6,4-7,5)$ & & 1,4 & $(1,1-1,6)$ & \\
\hline
\end{tabular}

${ }^{*} p<0,05,{ }^{* *} p<0,001$ 
Tabla 3. Riesgo de anemia en niños de dos a cinco meses atendidos en establecimientos de salud del Ministerio de Salud del Perú, 2012

\begin{tabular}{|c|c|c|c|c|c|c|}
\hline & OR Bivariado & (IC 95\%) & $p$ & OR Multivariable* & (IC 95\%) & $p$ \\
\hline \multicolumn{7}{|l|}{ Edad } \\
\hline 2 meses & 1,0 & & & 1,0 & & \\
\hline 3 meses & 1,0 & $(0,8-1,2)$ & 0,800 & 1,0 & $(0,9-1,3)$ & 0,728 \\
\hline 4 meses & 1,2 & $(0,9-1,4)$ & 0,099 & 1,2 & $(1,0-1,5)$ & 0,041 \\
\hline 5 meses & 1,2 & $(1,1-1,4)$ & 0,004 & 1,4 & $(1,2-1,6)$ & $<0,001$ \\
\hline \multicolumn{7}{|l|}{ Estado nutricional } \\
\hline Normal & 1,0 & & & 1,0 & & \\
\hline Desnutrición aguda & 1,1 & $(0,6-2,0)$ & 0,714 & 1,2 & $(0,7-2,2)$ & 0,564 \\
\hline Sobrepeso & 0,9 & $(0,8-1,1)$ & 0,399 & 0,9 & $(0,8-1,0)$ & 0,414 \\
\hline Obesidad & 0,7 & $(0,5-0,9)$ & 0,024 & 0,7 & $(0,5-1,0)$ & 0,034 \\
\hline \multicolumn{7}{|l|}{ Desnutrición crónica } \\
\hline No & 1,0 & & & 1,0 & & \\
\hline Sí & 1,2 & $(1,0-1,5)$ & 0,022 & 1,3 & $(1,0-1,6)$ & 0,025 \\
\hline \multicolumn{7}{|l|}{ Desnutrición global } \\
\hline No & 1,0 & & & 1,0 & & \\
\hline Sí & 1,3 & $(0,8-1,9)$ & 0,269 & 1,1 & $(0,8-1,8)$ & 0,602 \\
\hline
\end{tabular}

*Ajustado por sexo, altitud y región de residencia

La frecuencia de desnutrición crónica fue de 6,9\% (IC 95\%: 6,4-7,5) siendo más frecuente en lactantes de sexo masculino (8,2\%). Conforme es mayor la altitud sobre el nivel del mar, mayor es la frecuencia de desnutrición crónica $(p<0,001)$. Las regiones de Callao, Ancash y Junín presentaron las mayores frecuencias de desnutrición crónica en lactantes (Tabla 2).

La frecuencia global de desnutrición en lactantes fue de 1,4\% (IC 95\%: 1,1-1,6). No hubo diferencias en cuanto a los meses y sexo. De acuerdo a la altitud, la desnutrición global fue más frecuente entre los 4001 a 4801 metros de altitud (4,6\%). La región de Ayacucho presentó la mayor frecuencia de desnutrición global $(3,2 \%)$ (Tabla 2$)$.

En el análisis multivariable se hallaron como variables asociadas con tener anemia las edades de 4 (OR 1,2; IC 95\%: 1,0-1,5) y 5 meses (OR 1,4; IC 95\%: 1,2-1,6), presentar obesidad (OR 0,7; IC 95\%: 0,5-1,0) y presentar desnutrición crónica (OR 1,3; IC 95\%: 1,0-1,6) (Tabla 3).

\section{DISCUSIÓN}

Los resultados indican que la anemia en lactantes atendidos en establecimientos del Ministerio de Salud está presente desde antes de los primeros 6 meses de vida, y que los lactantes formarán parte de los anémicos moderados a los 6 meses de edad si no se realizan acciones para revertirlo.
En el presente estudio, el $25 \%$ de lactantes evaluados serían anémicos. Usando valores similares en Brasil, Guerreiro et al. (12) encontraron $20,2 \%$ de anemia en lactantes de 3 a 5 meses, mientras que en Argentina, Lancelli et al. (2), encontraron $20,6 \%$ en lactantes de 4 meses y de $30 \%$ en lactantes de cinco meses, con una prevalencia total entre ambos de $28,9 \%$. Sin embargo, usando valores de $\mathrm{Hb}$ menores a $11 \mathrm{~g} / \mathrm{dL}$ utilizado en otros estudios, como por ejemplo en Honduras, Zelaya et al. (3) encontraron $60 \%$ de anemia en lactantes entre los 4 a 6 meses; los resultados del presente estudio llegarían a valores cercanos a la mitad de lactantes con anemia.

En condiciones ideales, los niños nacidos a término tienen reservas de hierro que cubren sus demandas por aproximadamente cuatro meses ${ }^{(9)}$. Sin embargo, la $\mathrm{DH}$ en el lactante inicia con el problema de anemia o $\mathrm{DH}$ en las gestantes, entre varias condiciones que no permiten alcanzar el hierro corporal necesario en el recién nacido ${ }^{(16)}$ para así evitar la $\mathrm{DH}$ o anemia antes de los 6 meses de edad ${ }^{(17)}$. De acuerdo con la ENDES del $2012^{(7)}$, y también lo reportado por el SIEN ${ }^{(14)}$, casi un tercio de las gestantes presentó anemia, y si se agrega que por cada caso de anemia hay dos casos de $\mathrm{DH}$ sin anemia ${ }^{(1,17)}$, cerca del $90 \%$ de las gestantes presenta $\mathrm{DH}$ (con o sin anemia). En estas condiciones se producen los partos, provocando altas prevalencias de anemia por $\mathrm{DH}$ durante el primer año de vida ${ }^{(18-20)}$. El problema de anemia y la $\mathrm{DH}$ en la gestante, repercute 
en los depósitos de hierro del recién nacido ${ }^{(18-20)}$. Por lo que el abordaje de la anemia, especialmente del menor de un año, debe incluir, además, esfuerzos sustanciales de reducción de la anemia materna, asegurando el consumo del hierro medicado, como suplemento o tratamiento en la gestante, y reducir el abandono de la suplementación.

Para reducir la anemia en lactantes, se debería iniciar la suplementación con hierro, entre los dos a cuatro meses de edad, especialmente en los nacidos de madres anémicas, así como fomentar el clampaje tardío del cordón umbilical para mejorar los depósitos de hierro ${ }^{(21-22)}$. Se debe realizar un seguimiento a los recién nacidos de madres anémicas, e incluir la evaluación de hemoglobina antes de los 6 meses de edad. La suplementación con hierro en las gestantes, más la fortificación de alimentos, estrategias para el cambio de comportamiento, capacitación del personal, participación de promotores de salud y organismos no gubernamentales, mejoras en salud reproductiva y un mejor sistema de monitoreo, podrían disminuir de manera sustancial la proporción de niños con anemia a los 6 meses de edad, como se ha realizado en países como Nicaragua ${ }^{(23)}$, Tailandia ${ }^{(24)}$ y Nepal ${ }^{(25)}$.

Es preocupante observar desnutrición crónica a edades tempranas, una publicación de la Organización Panamericana de la Salud (OPS) ${ }^{(26)}$ en el 2008, mostró que la proporción de desnutridos crónicos en menores de 6 meses en el Perú era solo inferior que Haití $(18,6 \%)$ y Guatemala $(22,6 \%)$. En el presente estudio se encontró en desnutrición global, una proporción muy por debajo de la cifra encontrada por la OPS ${ }^{(26)}$ para el Perú en 2004-2008 (3,9\%). Respecto a la desnutrición aguda, es mucho menor a lo encontrado por la OPS $(2,9 \%)$, a pesar que el Perú mostró la menor cifra comparado a los demás países de la región ${ }^{(26)}$.

Las zonas rurales, y las de mayor altitud de la sierra del Perú siguen siendo las más afectadas pues presentan altas frecuencias para desnutrición crónica, en especial en residentes en altitudes mayores a los 3000 metros. Otras regiones en Latinoamérica entre los 2800 a 4100 metros de altitud como Potosí, en Bolivia, presentaron proporciones más altas de desnutrición crónica $(38,0 \%)^{(27)}$. Caritas del Perú (28) encontró en la región de Ancash, durante los años 2007-2010, un 37\% de desnutrición crónica en menores de 3 años, se logró una reducción al $27 \%$ con mejoras en la alimentación y tecnologías sencillas para conservación y manipulación de alimentos, entre otras.
Se encontró una asociación entre desnutrición crónica y anemia. Sin embargo, estos hallazgos requieren realizar mayores estudios, que por las características tiene un poder explicativo muy limitado. La anemia en el desnutrido es un evento relativamente frecuente, cuya etiología es multifactorial. Olivares M. et al. (29) encontraron una frecuencia del $53 \%$ de anemia en lactantes marásmicos, entre los 6 y 21 meses de edad, siendo un $30 \%$ de ellos por $\mathrm{DH}$, hallando una correlación directa con el puntaje $z$ de P/E y T/E.

Respecto a las limitaciones en el estudio, la distribución de la edad es hacia los cinco meses, debido a que los niños menores de un año son controlados en forma mensual. Esto se debe a que en el Perú se toma la muestra de $\mathrm{Hb}$ a partir de los seis meses, y explica por qué una amplísima mayoría no tiene resultados de hemoglobina, pero la fuente analizada ha permitido tener niños menores de 6 meses con estos datos. Por otro lado, los criterios para realizar la prueba de hemoglobina en esta edad no se conocen. Estos criterios podrían sesgar los resultados de la investigación, sin embargo, las cifras de hemoglobina han sido heterogéneas. Asimismo, por ser una fuente secundaria no se tiene datos como peso al nacer, patología al nacer, tipo de lactancia, frecuencia de enfermedades infecciosas, entre otras, así como variables de la madre relacionados a tipo de parto, $\mathrm{Hb}$ prenatal y/o preparto, suplemento o tratamiento con hierro, edad y datos socioeconómicos, que permitirían dar un mejor acercamiento al problema.

En conclusión se logró determinar la presencia de anemia y desnutrición crónica en los lactantes y con diferencias según edad, altitud, regiones de residencia y tipo de establecimiento de salud, y en desnutrición crónica incluso según sexo. Se encontró, además, mayor probabilidad de anemia en desnutridos crónicos.

Agradecimientos: al licenciado Juan Carlos Barboza, al ingeniero Manuel Prado Merino y al técnico Carlos Cosser Gamarra, miembros del equipo del Sistema de Información y Estado Nutricional (SIEN) de la Dirección Ejecutiva de Vigilancia Alimentaria y Nutricional (DEVAN) del Centro Nacional de Alimentación y Nutrición (CENAN) del Instituto Nacional de Salud (INS).

Contribuciones de autoría: GG participó en la concepción y diseño del estudio, análisis e interpretación de los datos, redacción del artículo, revisión crítica del artículo y asesoría estadística. OM participo en la concepción y diseño del artículo, análisis e interpretación de datos, revisión crítica del artículo y aprobación de su versión final.

Conflictos de interés: los autores declaran no tener conflictos de interés.

Fuentes de financiamiento: autofinanciado. 


\section{REFERENCIAS BIBLIOGRÁFICAS}

1. World Health Organization. Iron deficiency anemia assessment, prevention and control: a Guide for Programme Managers. Geneva: WHO; 2001.

2. Ianicelli J, Varea A, Falivene M, Disalvo L, Apezteguía M, González $H$. Prevalencia de anemia en lactantes menores de 6 meses asistidos en un centro de atención primaria de la ciudad de La Plata. Arch Argent Pediatr. 2012;110(2):120-5

3. Dewey KG, Brown KH. Update on technical issues concerning complementary feeding of young children in developing countries and implications for intervention programs. Food Nutr Bull. 2003 Mar;24(1):5-28.

4. Zelaya-Lozano E, Peña-Hernández A, París-Domínguez I, Madrid-Figueroa J. Anemia y reservas de hierro en niños menores de 24 meses aparentemente sanos. Honduras Pediatrica. 1997 AbrJun;18(2):38-41.

5. Rivera F, Walter T. Efecto de la anemia ferropriva en el lactante sobre el desarrollo psicológico del escolar. Rev Chil Pediatr. 1996;67(4);141-7.

6. Ceriani J, Carroli G, Pellegrini L, Ferreira M, Ricci C, Casas O, et al. Efecto del clampeo demorado del cordón umbilical en la ferritina sérica a los seis meses de vida. Estudio clínico controlado aleatorizado. Arch Argent Pediatr. 2010;108(3):201-8.

7. Instituto Nacional de Estadística e Informática. Encuesta Demográfica y de Salud Familiar 2012 Nacional y Departamental. Lima: INEI; 2013.

8. Organización Panamericana de la Salud. La Desnutrición en Lactantes y Niños Pequeños en América Latina y El Caribe: Alcanzando los Objetivos de Desarrollo del Milenio. Washington, DC: OPS; 2008.

9. Vega L. El encuentro cotidiano con la deficiencia de hierro en los lactantes. Rev Mex Pediatr. 1998 JulAgo;65(4):150-1.

10. Organización Mundial de la Salud. Concentraciones de hemoglobina para diagnosticar la anemia y evaluar su gravedad. Ginebra: OMS; 2011.

11. Ministerio de Salud, Instituto Nacional de Salud. Guía Técnica: Procedimiento para la determinación dela hemoglobina mediante hemoglobinómetro portátil. Lima: MINSA, INS; 2013.

12. Guerreiro dos Reis M, Spanó A, Aparecida I, Azevedo F, Bistafa M. La prevalencia de anemia en niños de 3 a 12 meses de vida en un servicio de salud de Ribeirão Preto, SP, Brasil. Rev Lat-Am Enferm. 2010 Jul-Ago;18(4):792-9.

13. Ministerio de Salud. DIRECTIVA SANITARIA No 050-MINSA/ DGSP-V.01. Directiva Sanitaria que establece la Suplementación Preventiva con Hierro en las Niñas y Niños menores de tres años. Lima: MINSA; 2012.

14. Munares-García O, Gómez-Guizado G, Barboza-Del Carpio J, SánchezAbanto J. Niveles de hemoglobina en gestantes atendidas en establecimientos del Ministerio de Salud del Perú, 2011. Rev Peru Med Exp Salud Publica. 2012;29(3):329-36.

15. Ministerio de Salud. Norma Técnica de Salud para el Control del Crecimiento y Desarrollo de la Niña y el Niño Menor de Cinco Años. Lima: MINSA; 2011.

16. Baker R, Greer F, the Committee on Nutrition. Clinical Report - Diagnosis and prevention of iron deficiency and iron-deficiency anemia in infants and young children $(0-3$ year of age $)$. Pediatrics. 2010 Nov;126(5):1-10.

17. Lutter CK. Iron deficiency in young children in low-income countries and new approaches for its prevention. J Nutr. 2008 Dec;138(12):2523-8. doi: 10.3945/jn.108.095406.

18. Colomer J, Colomer C, Gutiérrez D, Jubert A, Nolasco A, Donat J, et al. Anaemia during pregnancy as a risk factor for infant iron deficiency: report from the Valencia Infant Anaemia Cohort (VIAC) study. Paediatr Perinat Epidemiol. 1990 Apr;4(2):196-204.

19. Olivares M, Walter T. Consecuencias de la deficiencia de hierro. Rev Chil Nutr. 2003 Dic;30(3):226-33.

20. Chaparro CM.Setting the stagefor child health and development: prevention of iron deficiency in early infancy. J Nutr. 2008 Dec;138(12):2529-33.

21. McDonald SJ, Middleton P, Dowswell T, Morris PS. Effect of timing of umbilical cord clamping of term infants on maternal and neonatal outcomes. Cochrane Database Syst
Rev. 2013 Jul 11;7:CD004074. doi: 10.1002/14651858.CD004074.pub3.

22. Venâncio SI, Bertazzi R, Dias SR, Mondini L, Goi MC, Lum S. Efeitos do clampeamento tardio do cordão umbilical sobre os níveis de hemoglobina e ferritina em lactentes aos três meses de vida. Cad Saude Publica. 2008;24(suppl.2):S323-S331.

23. Mora J. Integrated Anemia Control Strategy (IACS) has significantly reduced anemia in women and children in Nicaragua. Ottawa: Micronutrient Initiative; 2007.

24. Winichagoon P. Prevention and control of anemia: Thailand experiences. J Nutr. 2002 Apr;132(4 Suppl):862S-6S.

25. Pokharel RK, Maharjan MR, Mathema P, Harvey PWJ. Success in Delivering Interventions to Reduce Maternal Anemia in Nepal: A Case Study of the Intensification of Maternal and Neonatal Micronutrient Program. Washington, DC: The USAID Micronutrient and Child Blindness Project; 2011.

26. Organización Panamericana de la Salud. La Desnutrición en lactantes y niños pequeños en América Latina y El Caribe: Alcanzando los objetivos de desarrollo del milenio. Washington, DC: OPS; 2008.

27. Cruz-Agudo Y, Jones AD, Berti PR, Larrea-Macías S. Lactancia materna, alimentación complementaria y malnutrición infantil en los Andes de Bolivia. Arch Latinoam Nutr. 2010;60(1):7-14.

28. Caritas del Perú, Ally Micuy. Reducción de la desnutrición crónica y la anemia infantil en Ancash. Proyecto Ally Micuy 2007-2010. Lima: Studio Digital Editores; 2010.

29. Olivares M, Hertrampf E, Chadud P. Anemia del lactante desnutrido marásmico. Rev Chil Pediatr. 1992 Ago;63(4):191-5.

Correspondencia: Guillermo Gómez
Guisado

Dirección: Av. Tizón y Bueno 276 Jesús

María. Lima, Perú

Teléfono: 6176200 Anexo 6605

Correo electrónico:ggomez@ins.gob.pe 